\title{
Monitoring biopolymer degradation by Taylor
}

\section{dispersion analysis}

\author{
Joseph Chamieh ${ }^{1}$, Jean Philippe Biron ${ }^{1}$, Luca Cipelletti ${ }^{2}$, Hervé Cottet ${ }^{* 1}$ \\ ${ }^{1}$ Institut des Biomolécules Max Mousseron (IBMM, UMR 5247 CNRS, Université de \\ Montpellier, Ecole Nationale Supérieure de Chimie de Montpellier), Campus Triolet, Place \\ Eugène Bataillon, CC 1706, 34095 Montpellier Cedex 5, France \\ ${ }^{2}$ Laboratoire Charles Coulomb (L2C), UMR 5221 CNRS, Université de Montpellier, \\ Montpellier, F-France.
}

\section{Table of contents}

- Figure S1. Variation of the hydrodynamic radii as function of the degradation time of the three populations obtained by deconvolution of the taylorgrams with three Gaussian peaks for G5 hydrolytic degradation

- Figure S2. Variation of the proportions of the three populations, obtained by deconvolution of the taylorgrams with three Gaussian peaks for G5 hydrolytic degradation, as function of the degradation time. 


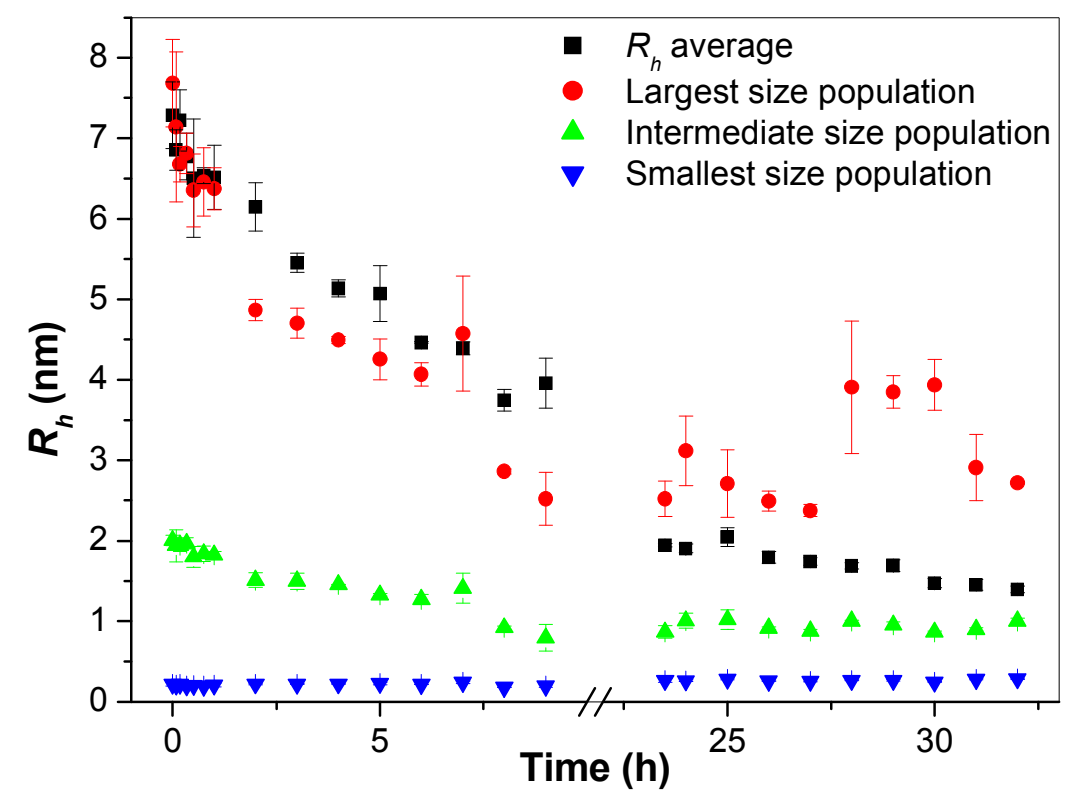

Figure S1. Variation of the hydrodynamic radii as function of the degradation time of the three populations obtained by deconvolution of the taylorgrams with three Gaussian peaks for G5 hydrolytic degradation. Error bars are the standard deviations on three repetitions. Experimental conditions as in Figure 1.

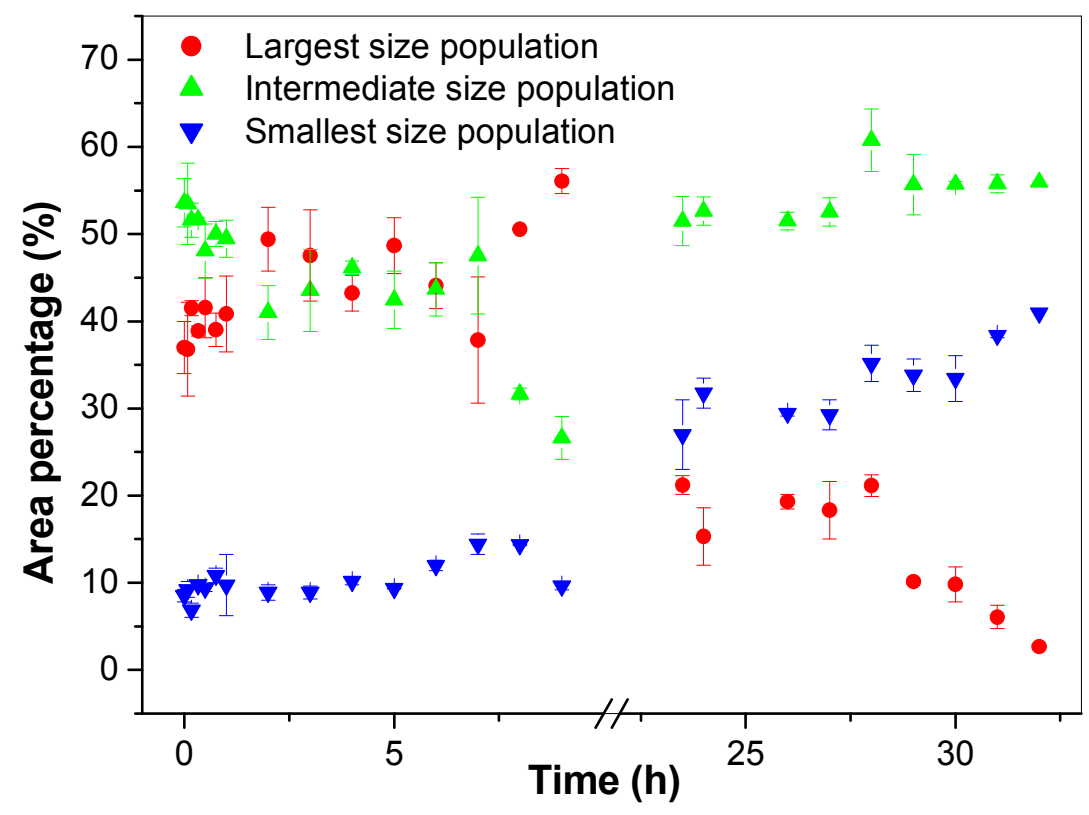

Figure S2. Variation of the proportions of the three populations, obtained by deconvolution of the taylorgrams with three Gaussian peaks for G5 hydrolytic degradation, as function of the degradation time. Error bars are the standard deviations on three repetitions. Experimental conditions as in Figure 1. 